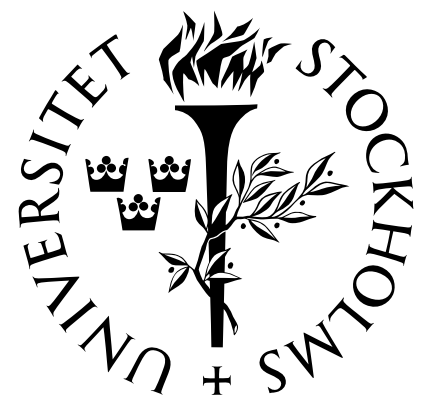

\title{
On vertex conditions for elastic systems
}

\author{
Jean-Claude Kiik, Pavel Kurasov and \\ Muhammad Usman
}

Research Reports in Mathematics

Number 1, 2015

Department of MATHEMATics

STOCKHOLM UNIVERSITY 
Electronic version of this document is available at http://www.math.su.se/reports/2015/1

Date of publication: January 10, 2015.

2010 Mathematics Subject Classification:

Primary 34L25, 81U40; Secondary 35P25, 81V99.

Keywords: Fourth-order differential operator, Matching conditions, Elasticity.

Postal address:

Department of Mathematics

Stockholm University

S-106 91 Stockholm

Sweden

Electronic addresses:

http://www.math.su.se/

info@math.su.se 


\title{
On vertex conditions for elastic systems
}

\author{
Jean-Claude Kiik, Pavel Kurasov, and Muhammad Usman \\ Department of Mathematics, Stockholm University, \\ SE-10691, Stockholm, Sweden
}

January 10, 2015

\section{Corresponding author:}

Pavel Kurasov, kurasov@ math.su.se, phone +46-8164544, fax +46-86126717

\begin{abstract}
In this paper vertex conditions for the fourth order differential operator on the simplest metric graph - the $Y$-graph, - are discussed. In order to make the operator symmetric one needs to impose extra conditions on the limit values of functions and their derivatives at the central vertex. It is shown that such conditions corresponding to the free movement of beams depend on the angles between the beams in the equilibrium position.
\end{abstract}

\section{Keywords:}

Fourth-order differential operator, Matching conditions, Elasticity.

\section{Motivation}

Quantum graphs - differential operators on metric graphs - form a well-established formalism to model wave propagation in thin channels and other physical systems where interesting phenomena occur in a neighborhood of a system of lowdimensional manifolds [BK, K, P]. It appears that ordinary differential equations on graph edges coupled by certain vertex conditions give a rather good approximation of wave propagation in thin domains. Intensive research was devoted to understanding which particular vertex conditions give the best approximation. One may expect that the geometry (first of all angles between the channels) of the vertex should be reflected in these conditions. To study this question it is enough to consider the star graph and in this article we are going to restrict our consideration to the star graph $\Gamma$ formed by 3 semi infinite edges $\left[x_{j}, \infty\right), j=1,2,3$. There is just 
one vertex $V=\left\{x_{1}, x_{2}, x_{3}\right\}$. It appears that to approximate the wave equation in an $\epsilon$-neighborhood of $\Gamma$ one should consider the wave equation on $\Gamma$ subject to either by standard vertex conditions

$$
\left\{\begin{array}{l}
u \text { is continuous at the vertex } V ; \\
\sum_{x_{j} \in V} u^{\prime}\left(x_{j}\right)=0
\end{array}\right.
$$

or Dirichlet conditions

$$
u\left(x_{j}\right)=0, x_{j} \in V_{m} .
$$

Vertex conditions of the second type lead to independent edges, hence only conditions of the first type are of interest. It appears that the geometry of the junction is not reflected in the vertex conditions at all. In what follows right now we shall try to explain the reason for this phenomenon.

In physics it is common to use quadratic forms instead of operators, since often it is easier to check that a quadratic (sesquilinear) form is semibounded and closed instead of proving that an operator is self-adjoint. The quadratic form corresponding to the Laplacian on $\Gamma$ is given by the integral of $\left|u^{\prime}(x)\right|^{2}$ plus possibly an additional term coming from the vertex. This additional term describes the interaction between the waves and the vertex and should be absent if one is looking for conditions corresponding to the free motion. We end up with the quadratic form

$$
Q[u, v]=\sum_{j=1}^{3} \int_{x_{j}}^{\infty} u^{\prime}(x) \overline{v^{\prime}(x)} d x
$$

This form is positive and closed on the set of functions from the Sobolev space $W_{2}^{1}(\Gamma \backslash V)=\oplus \sum_{j=1}^{3} W_{2}^{1}\left[x_{j}, \infty\right)$. If no further conditions at the vertex are assumed, then the corresponding self-adjoint operator is the Laplace operator $L_{I I}=$ $-\frac{d^{2}}{d x^{2}}$ defined on the functions from $W_{2}^{2}(\Gamma \backslash V)$ satisfying Neumann conditions $u^{\prime}\left(x_{j}\right)=0$. The three edges are again independent of each other. Therefore the only way to introduce coupling between the edges is through additional vertex conditions. Since the functions from $W_{2}^{1}$ are continuous, but their derivates are not necessarily continuous, these vertex conditions may involve only the values of the function at the end points $u\left(x_{j}\right)$. Since every solution to a partial differential equation in the thick domain is continuous at the origin, it is natural to impose the continuity condition

$$
u\left(x_{1}\right)=u\left(x_{2}\right)=u\left(x_{3}\right) .
$$

The self-adjoint operator corresponding to the quadratic form (1.3) defined on functions from $W_{2}^{1}(\Gamma \backslash V)$ satisfying (1.4) is precisely the Laplace operator $L_{I I}$ with the domain given by standard conditions (1.1). 
We have seen that vertex conditions corresponding to free motion do not contain any information about the geometry of the junction, especially the angles between the edges are not reflected. In what follows we turn to problems of elasticity and will show how the geometry of the junction is reflected in vertex conditions corresponding to free motion.

\section{Elasticity}

Elasticity problems are usually described by fourth order differential operators [LL]. Consider therefore the differential operator $L_{I V}:=\frac{d^{4}}{d x^{4}}$ first on the interval $[0, \infty)$ and later on the graph $\Gamma$. The corresponding wave equation

$$
\frac{\partial^{4}}{\partial x^{4}} u=-\frac{\partial^{2}}{\partial t^{2}} u
$$

can be used to describe the dynamics of small deflection of beams. Thus the operator $L_{I V}$ in $L^{2}(0, \infty)$ corresponds to a single long beam. The operator can be made self-adjoint by choosing appropriate boundary conditions at the origin. For example, in the case when the end point of the beam is clamped the corresponding self-adjoint boundary condition is

$$
u(0)=u^{\prime}(0)=0 .
$$

And in the case when the end point is free we can impose the following condition to make the operator self-adjoint

$$
u^{\prime \prime}(0)=u^{\prime \prime \prime}(0)=0
$$

One can describe all possible boundary conditions at the end point $x=0$ by the following zero, one, two and three-parameters boundary conditions, respectively (see $[\mathrm{Y}])$ :

0) $u(0)=u^{\prime}(0)=0$,

i) $u(0)=0, \quad u^{\prime \prime}(0)=\gamma_{1} u^{\prime}(0)$,

ii) $u^{\prime}(0)=\gamma_{3} u(0), \quad-u^{\prime \prime \prime}(0)+\overline{\gamma_{3}} u^{\prime \prime}(0)=\gamma_{2} u(0)$,

iii) $\left\{\begin{array}{l}u^{\prime \prime}(0)=\gamma_{3} u(0)+\gamma_{1} u^{\prime}(0) \\ u^{\prime \prime \prime}(0)=-\gamma_{2} u(0)-\overline{\gamma_{3}} u^{\prime}(0),\end{array}\right.$

where $\gamma_{1}, \gamma_{2}$ are arbitrary real numbers and $\gamma_{3}$ is an arbitrary complex number. This formalism can be carried out to the case where several beams are connected 
together at one junction. It is not difficult to describe all possible vertex conditions leading to self-adjoint operators. To describe all such vertex conditions one may use either von Neumann theory [RS], boundary triples [DM] or scattering matrix approach $[\mathrm{K}, \mathrm{KE}, \mathrm{KN}]$. Our goal here is not to carry out such description, but to derive conditions corresponding to the free dynamics of beams.

Consider first the case where just two beams are connected. Assume that the equilibrium position corresponds to the case when the two beams form a line. Such beams may be parameterized as $\left(-\infty, x_{0}\right]$, and $\left[x_{0}, \infty\right)$. The quadratic form corresponding to the free motion is given by

$$
Q[u, v]=\int_{-\infty}^{\infty} u^{\prime \prime}(x) \overline{v^{\prime \prime}(x)} d x .
$$

First of all the function describing the deflection should be continuous, otherwise the beams are not connected at $x_{0}$

$$
u\left(x_{0}-0\right)=u\left(x_{0}+0\right) .
$$

The beams are free, no external force is applied if the graph of $u(x)$ does not form any angle at $x_{0}$, i.e. the first derivative is continuous

$$
u^{\prime}\left(x_{0}-0\right)=u^{\prime}\left(x_{0}+0\right) .
$$

The quadratic form on the domain of functions from $W_{2}^{2}\left(\mathbb{R} \backslash\left\{x_{0}\right\}\right)$ satisfying conditions (2.3) and (2.4) is semi bounded and closed. Let us calculate the self-adjoint operator corresponding to this quadratic form. First of all one needs to determine the set of all $u$, for which the quadratic form $Q[u, v]$ is a bounded liner functional with respect to $v$. Taking $v$ from $C_{0}^{\infty}\left(\mathbb{R} \backslash\left\{x_{0}\right\}\right)$ we see that the second derivative of the function $u^{\prime \prime}$ should be from $L_{2, \text { loc }}\left(\mathbb{R} \backslash\left\{x_{0}\right\}\right)$. In other words $u \in W_{2}^{4}\left(\mathbb{R} \backslash\left\{x_{0}\right\}\right)$ and one may carry out integration by parts:

$$
\begin{aligned}
Q[u, v]= & \int_{-\infty}^{x_{0}} u^{(i v)}(x) \overline{v(x)} d x+\int_{x_{0}}^{-\infty} u^{(i v)}(x) \overline{v(x)} d x \\
& -u^{\prime \prime}\left(x_{0}+0\right) \overline{v^{\prime}}\left(x_{0}+0\right)+u^{\prime \prime \prime}\left(x_{0}+0\right) \bar{v}\left(x_{0}+0\right) \\
& +u^{\prime \prime}\left(x_{0}-0\right) \overline{v^{\prime}}\left(x_{0}-0\right)-u^{\prime \prime \prime}\left(x_{0}-0\right) \bar{v}\left(x_{0}-0\right) \\
= & \int_{-\infty}^{x_{0}} u^{(i v)}(x) \overline{v(x)} d x+\int_{x_{0}}^{-\infty} u^{(i v)}(x) \overline{v(x)} d x \\
& -\left(u^{\prime \prime}\left(x_{0}+0\right)-u\left(x_{0}-0\right)\right) \overline{v^{\prime}}\left(x_{0}\right)+\left(u^{\prime \prime \prime}\left(x_{0}+0\right)-u^{\prime \prime \prime}\left(x_{0}-0\right)\right) \bar{v}\left(x_{0}\right),
\end{aligned}
$$

where on the last step we used that the function $v$ satisfies (2.3) and (2.4). The internal terms are bounded linear functionals with respect to $v$, while the functionals

$$
v \mapsto v\left(x_{0}\right) \text { and } v \mapsto v^{\prime}\left(x_{0}\right)
$$


are not. It follows that the coefficients in front of these functionals must be equal to zero

$$
\left\{\begin{array}{l}
u^{\prime \prime}\left(x_{0}-0\right)=u^{\prime \prime}\left(x_{0}+0\right), \\
u^{\prime \prime \prime}\left(x_{0}-0\right)=u^{\prime \prime \prime}\left(x_{0}+0\right) .
\end{array}\right.
$$

The operator $\frac{d^{4}}{d x^{4}}$ defined on the set of functions from $W_{2}^{4}\left(\mathbb{R} \backslash\left\{x_{0}\right\}\right)$ satisfying matching conditions (2.3), (2.4) and (2.6) is the self-adjoint operator corresponding to the quadratic form. As in the case of Laplacian the vertex $x_{0}$ can be removed and the two edges may substituted by one edge $(-\infty, \infty)$.

Our goal is to understand how the free matching conditions (2.3), (2.4), (2.6) can be generalized to the case when several beams are joined at one vertex, having different angles in-between in the equilibrium position. The function $u$ describing small deflections of the system from the equilibrium can be considered as a function on the graph $\Gamma$ made up of three half-lines connected at zero and making angles $\alpha, \beta$ and $\gamma$, none of which are equal to 0 or $\pi$ (see Figure 1 ). It will be convenient to denote by $u_{j}$ the values of the function $u$ on the corresponding edges. The Hilbert space is $L_{2}(\Gamma):=\bigoplus_{i=1}^{3} L_{2}[0, \infty)$ and we are interested in free vertex conditions for the operator of fourth differentiation $L_{I V}=\frac{d^{4}}{d x^{4}}$.

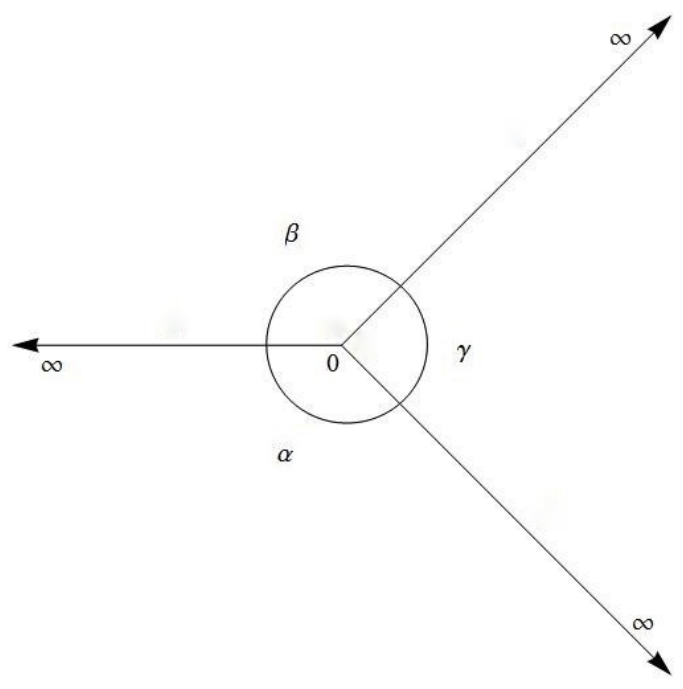

Figure 1: A Y-graph with certain angles between the branches.

The literature devoted to this problem is rather limited especially using rigorous mathematical language. We have found the articles [DN1, DN2], where the following two types of vertex conditions are considered without giving much motivation 
(equations (4)-(8) from [DN2] and (2)-(5) from [DN1] adopted to our notations)

$$
\left\{\begin{array} { l } 
{ u _ { 1 } ( 0 ) = u _ { 2 } ( 0 ) = u _ { 3 } ( 0 ) , } \\
{ u _ { 1 } ^ { \prime } ( 0 ) = u _ { 2 } ^ { \prime } ( 0 ) = u _ { 3 } ^ { \prime } ( 0 ) , } \\
{ u _ { 1 } ^ { \prime \prime } ( 0 ) + u _ { 2 } ^ { \prime \prime } ( 0 ) + u _ { 3 } ^ { \prime \prime } ( 0 ) = 0 , } \\
{ u _ { 1 } ^ { \prime \prime \prime } ( 0 ) + u _ { 2 } ^ { \prime \prime \prime } ( 0 ) + u _ { 3 } ^ { \prime \prime \prime } ( 0 ) = 0 , }
\end{array} \quad \text { and } \quad \left\{\begin{array}{l}
u_{1}(0)=u_{2}(0)=u_{3}(0), \\
u_{1}^{\prime}(0)+u_{2}^{\prime}(0)+u_{3}^{\prime}(0)=0, \\
u_{1}^{\prime \prime}(0)=u_{2}^{\prime \prime}(0)=u_{3}^{\prime \prime}(0), \\
u_{1}^{\prime \prime \prime}(0)+u_{2}^{\prime \prime \prime}(0)+u_{3}^{\prime \prime \prime}(0)=0 .
\end{array}\right.\right.
$$

Probably the main motivation to use these vertex conditions is just the analogy with the standard conditions (1.1) widely used for the second order differential operators. Another type of vertex conditions is used in [M] (equations (1.3.16), (1.3.17), (1.3.19) and (1.3.21) again adopted to our notations and changing the number of joined together beams from 4 to 3 )

$$
\left\{\begin{array}{l}
u_{1}(0)=u_{2}(0)=u_{3}(0) \\
-u_{1}^{\prime}(0)=u_{2}^{\prime}(0)=u_{3}^{\prime}(0) \\
-u_{1}^{\prime \prime}(0)+u_{2}^{\prime \prime}(0)+u_{3}^{\prime \prime}(0)=0 \\
u_{1}^{\prime \prime \prime}(0)+u_{2}^{\prime \prime \prime}(0)+u_{3}^{\prime \prime \prime}(0)=0
\end{array}\right.
$$

As it will be shown later, these vertex conditions correspond to the case where all three beams are parallel in the equilibrium position. If the beams in the equilibrium lie in the plane, but form arbitrary angles in-between, then one should use different free conditions. derivation of these conditions is the main goal of our paper.

It appears that the angles between the beams make their way into the free matching conditions in contrast to the case of second order differential operator. Our main result of this paper is the description of the free matching conditions for the fourth order differential operator.

Theorem 1 The free system of three plane beams having angles $\alpha, \beta$, and $\gamma$ in the equilibrium position (see Figute 1) is described by the fourth order differential operator $L_{I V}$, on the domain of functions from the Sobolev space $W_{2}^{4}(\Gamma \backslash\{V\}):=$ $\bigoplus_{i=1}^{3} W_{2}^{4}(0, \infty)$ satisfying the following vertex conditions

$$
\left\{\begin{array}{l}
u_{1}(0)=u_{2}(0)=u_{3}(0) \\
\sin \alpha u_{1}^{\prime}(0)+\sin \beta u_{2}^{\prime}(0)+\sin \gamma u_{3}^{\prime}(0)=0 \\
\frac{u_{1}^{\prime \prime}(0)}{\sin \alpha}=\frac{u_{2}^{\prime \prime}(0)}{\sin \beta}=\frac{u_{3}^{\prime \prime}(0)}{\sin \gamma} \\
\sum_{j=1}^{3} u_{j}^{\prime \prime \prime}(0)=0
\end{array}\right.
$$

provided none of the angles $\alpha, \beta$ and $\gamma$ are equal to 0 or $\pi$. 
Note that it is not our intention to describe all possible self-adjoint vertex conditions for the differential operator $L_{I V}$. We are discussing the most natural vertex conditions. We expect that precisely these conditions appear if one consider approximation of the system by narrow channels.

\section{Derivation of the matching conditions}

We consider the system of three beams joined together at one point 0 as shown in Figure 1. We assume that in the equilibrium the beams are placed in a plane forming graph $\Gamma$ presented in Figure 1. Small displacements of the beams in the direction perpendicular to the plane are described by the function $u(x), x \in \Gamma$. If no external forces are applied to the system of beams, then at every point $x_{0} \in \Gamma$ there is a tangential plane to the graph of $u$. As we already seen if $x_{0}$ is an internal point on one of the edges, then the requirement that there exists a tangential plane (line) at $x_{0}$ leads to conditions (2.3), (2.4), and (2.6).

Our main goal now is to study the vertex of $\Gamma$ and matching conditions there. We require that there is a tangential plane to the graph $u$ even at the vertex. Such plane may exist only if the function $u$ is continuous there

$$
u_{1}(0)=u_{2}(0)=u_{3}(0)
$$

where the lower indices indicates the edges.

Another additional condition on the first derivative of functions is obtained by requiring that the tangential lines to the graphs of functions $u_{i}, i=1,2,3$ at the point $x=0$ lie in the same plane. To find the tangent vectors along each edge we assume the Y-graph is lying in $x y$-plane in $\mathbb{R}^{3}$ as shown in Figure 2. It is natural to choose one of the axis along one of the beams in equilibrium. Then, the tangent vectors $\vec{a}^{1}$, $\vec{a}^{2}$ and $\vec{a}^{3}$ to the graph of functions $u_{1}, u_{2}$ and $u_{3}$, respectively, are given by

$$
\begin{aligned}
& \vec{a}^{1}=\left(-\cos (\pi-\beta), \sin (\pi-\beta), u_{1}^{\prime}(0)\right)=\left(\cos \beta, \sin \beta, u_{1}^{\prime}(0)\right) \\
& \vec{a}^{2}=\left(-\cos (\pi-\alpha),-\sin (\pi-\alpha), u_{2}^{\prime}(0)\right)=\left(\cos \alpha,-\sin \alpha, u_{2}^{\prime}(0)\right) \\
& \vec{a}^{3}=\left(1,0, u_{3}^{\prime}(0)\right) .
\end{aligned}
$$

These three tangent vectors lie in the same plane if and only if the following determinant is equal to zero

$$
\begin{aligned}
& \operatorname{det}\left(\begin{array}{ccc}
\cos \beta & \sin \beta & u_{1}^{\prime}(0) \\
\cos \alpha & -\sin \alpha & u_{2}^{\prime}(0) \\
1 & 0 & u_{3}^{\prime}(0)
\end{array}\right) \\
= & \sin \alpha u_{1}^{\prime}(0)+\sin \beta u_{2}^{\prime}(0)-u_{3}^{\prime}(0)(\cos \beta \sin \alpha+\sin \beta \cos \alpha)=0 .
\end{aligned}
$$




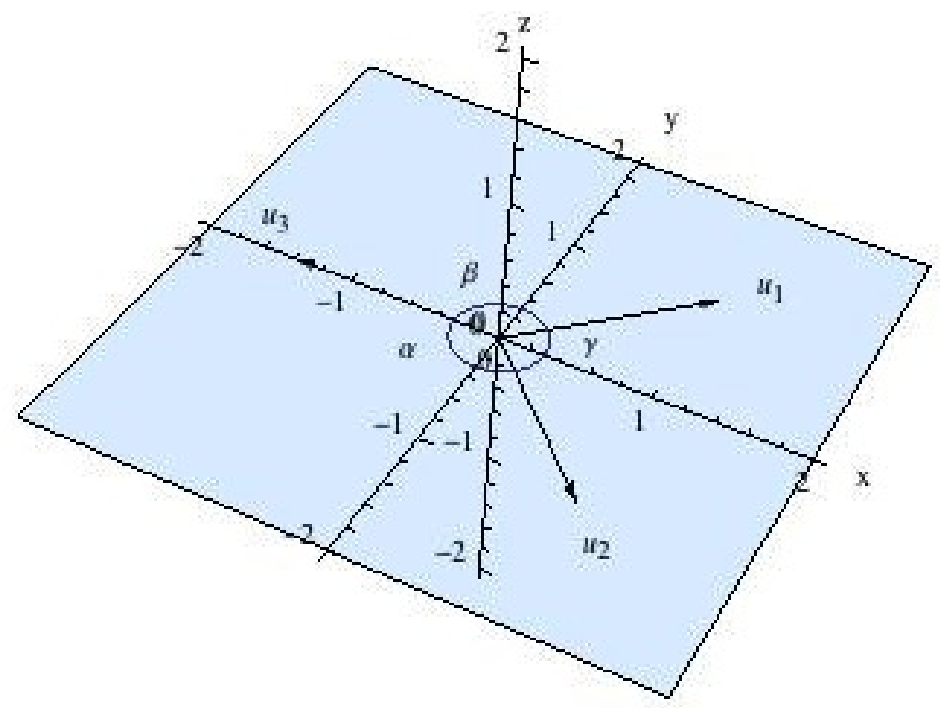

Figure 2: Y-graph in $\mathbb{R}^{3}$ with angles $\alpha, \beta$ and $\gamma$.

Using the trigonometric reduction $\sin \alpha \cos \beta+\cos \alpha \sin \beta=\sin (\alpha+\beta)$ we get the following matching condition

$$
\sin \alpha u_{1}^{\prime}(0)+\sin \beta u_{2}^{\prime}(0)-\sin (\alpha+\beta) u_{3}^{\prime}(0)=0 .
$$

Further, noting that $\sin (\alpha+\beta)=\sin (2 \pi-\gamma)=-\sin \gamma$ this condition can be written as

$$
\sin \alpha u_{1}^{\prime}(0)+\sin \beta u_{2}^{\prime}(0)+\sin \gamma u_{3}^{\prime}(0)=0 .
$$

Thus, so far we have the following three matching conditions

$$
\left\{\begin{array}{l}
u_{1}(0)=u_{2}(0)=u_{3}(0) \\
\sin \alpha u_{1}^{\prime}(0)+\sin \beta u_{2}^{\prime}(0)+\sin \gamma u_{3}^{\prime}(0)=0 .
\end{array}\right.
$$

Proof of Theorem 1. With the differential expression $\frac{d^{4}}{d x^{4}}$ one associates two differential operators: the maximal and the minimal ones. It is standard to define the minimal operator, $L^{\mathrm{min}}$, on smooth functions with the support separated from the vertex, that is:

$$
\operatorname{Dom}\left(L^{\mathrm{min}}\right)=C_{0}^{\infty}(\Gamma \backslash\{0\}):=\bigoplus_{i=1}^{3} C_{0}^{\infty}(0, \infty) .
$$

Using integration by parts, it is easy to see that the minimal operator is symmetric.

The maximal operator, $L^{\max }$, is defined on the domain of all functions from the Hilbert space $L^{2}(\Gamma)$ such that their images under the differential operator are still 
in the Hilbert space, i.e.

$$
\operatorname{Dom}\left(L^{\max }\right)=W_{2}^{4}(\Gamma \backslash\{0\})=\bigoplus_{i=1}^{3} W_{2}^{4}(0, \infty) .
$$

It consist of all square integrable functions having square integrable fourth derivatives. The maximal operator is not self-adjoint, or even symmetric.

Any self-adjoint operator corresponding to the graph $\Gamma$ should have a domain lying inside the domain of the maximal operator, but should definitely contain the domain of the minimal one. The differential operator $\frac{d^{4}}{d x^{4}}$ can be made self-adjoint by restriciting the maximal operator with the help of certain matching conditions at the vertex. It follows that the following inclusion for the domains of the minimal and maximal operators hold

$$
\operatorname{Dom}\left(L^{\min }\right) \subset \operatorname{Dom}(L) \subset \operatorname{Dom}\left(L^{\max }\right)
$$

where $L$ is any self-adjoint operator associated with $\frac{d^{4}}{d x^{4}}$.

Let us consider the quadratic form

$$
Q[u, v]=\sum_{i=1}^{3} \int_{0}^{\infty} u_{i}^{\prime \prime}(x) \overline{v_{i}^{\prime \prime}}(x) d x
$$

on the domain $W_{2}^{2}(\Gamma \backslash\{0\})$. Clearly, $Q[u, u] \geq 0$ and closed. Therefore, there exist a self-adjoint operator $L$ such that $Q[u, v]=\langle L u, v\rangle$, if $u \in \operatorname{Dom}(L)$ then by Rieszrepresentation theorem there exist a function $f$ in $L^{2}(\Gamma)$ such that $\langle L u, v\rangle=\langle f, v\rangle$ and $\langle f, v\rangle$ is a bounded linear functional with respect to $v$.

It is clear that every $u \in \operatorname{Dom}(L)$ is from $W_{2}^{4}(\Gamma \backslash\{0\})$. Integrating twice by parts the right hand side of (3.3) we get

$$
Q[u, v]=\sum_{i=1}^{3}\left(u_{i}^{\prime \prime \prime}(0) \overline{v_{i}}(0)-u_{i}^{\prime \prime}(0) \overline{v_{i}^{\prime}}(0)+\int_{0}^{\infty} u_{i}^{(i v)}(x) \overline{v_{i}}(x) d x\right)
$$

Now, using the conditions (3.2) for the functions $v_{i}, i=1,2,3$ and assuming $\alpha, \beta, \gamma \neq 0, \pi$ we arrive at

$$
\begin{aligned}
Q[u, v]=\left(\sum_{i=1}^{3} u_{i}^{\prime \prime \prime}(0)\right) & \overline{v_{1}}(0)+\left(u_{3}^{\prime \prime}(0) \frac{\sin \alpha}{\sin \gamma}-u_{1}^{\prime \prime}(0)\right) \overline{v_{1}^{\prime}}(0) \\
& +\left(u_{3}^{\prime \prime}(0) \frac{\sin \beta}{\sin \gamma}-u_{2}^{\prime \prime}(0)\right) \overline{v_{2}^{\prime}}(0)+\sum_{i=1}^{3} \int_{0}^{\infty} u_{i}^{(i v)} \overline{v_{i}} d x
\end{aligned}
$$


$Q[u, v]$ is a bounded linear functional with respect to $v$ if and only if the coefficients in front of $\overline{v_{1}}(0), \overline{v_{1}^{\prime}}(0)$ and $\overline{v_{2}^{\prime}}(0)$ are equal to 0 . This gives us the following three more conditions

$$
\left\{\begin{array}{l}
\sin \gamma u_{1}^{\prime \prime}(0)-\sin \alpha u_{3}^{\prime \prime}(0)=0 \\
\sin \gamma u_{2}^{\prime \prime}(0)-\sin \beta u_{3}^{\prime \prime}(0)=0 \\
\sum_{j=1}^{3} u_{j}^{\prime \prime \prime}(0)=0
\end{array}\right.
$$

The above set of conditions along with (3.2) make the operator $L_{I V}$ self-adjoint defined on the sobolev class $W_{2}^{4}(\Gamma \backslash\{0\})$. This completes the proof of Theorem 1.

\section{Special cases and examples}

Case when $\gamma=\pi$. In the proof of theorem 1 it was assumed that none of the angles $\alpha, \beta$ and $\gamma$ are equal to 0 or $\pi$. In the case when one of them is equal to zero our graph reduces to, rather less interesting case of, two half-lines.

When one of the three angles is equal to $\pi$ (let us say $\gamma=\pi$ ), the condition that the tangent vectors to the functions $u_{i}, i=1,2,3$ at $x=0$ lie in the same plane is equivalent to

$$
u_{1}^{\prime}(0)=-u_{2}^{\prime}(0) .
$$

Observe that we get no restriction on $u_{3}^{\prime}(0)$. Using this condition along with the continuity condition $u_{1}(0)=u_{2}(0)=u_{3}(0)$ into the quadratic form (3.4) for the functions $v_{i}, i=1,2,3$ we get

$$
Q[u, v]=\left(\sum_{i=1}^{3} u_{i}^{\prime \prime \prime}(0)\right) \overline{v_{1}}(0)-\left(u_{1}^{\prime \prime}(0)-u_{2}^{\prime \prime}(0)\right) \overline{v_{1}^{\prime}}(0)-u_{3}^{\prime \prime}(0) \overline{v_{3}^{\prime}}(0)+\sum_{i=1}^{3} \int_{0}^{\infty} u_{i}^{(i v)} \overline{v_{i}} d x .
$$

As before, it is a bounded linear functional with respect to $v$ if and only if the coefficients in front of $\overline{v_{1}}(0), \overline{v_{1}^{\prime}}(0)$ and $\overline{v_{3}^{\prime}}(0)$ are equal to 0 . This gives us the following set of conditions

$$
\left\{\begin{array}{l}
u_{1}(0)=u_{2}(0)=u_{3}(0), \\
u_{1}^{\prime}(0)=-u_{2}^{\prime}(0), \\
u_{1}^{\prime \prime}(0)=u_{2}^{\prime \prime}(0), \quad u_{3}^{\prime \prime}(0)=0, \\
\sum_{j=1}^{3} u_{j}^{\prime \prime \prime}(0)=0 .
\end{array}\right.
$$


Example 1. For the symmetric case when $\gamma=2 \delta$, with $\delta \neq 0, \pi$ and $\alpha=\beta=\pi-\delta$ our matching conditions read as

$$
\left\{\begin{array}{l}
u_{1}(0)=u_{2}(0)=u_{3}(0) \\
u_{1}^{\prime}(0)+u_{2}^{\prime}(0)+2 \cos \delta u_{3}^{\prime}(0)=0 \\
2 \cos \delta u_{1}^{\prime \prime}(0)=2 \cos \delta u_{2}^{\prime \prime}(0)=u_{3}^{\prime \prime}(0) \\
\sum_{j=1}^{3} u_{j}^{\prime \prime \prime}(0)=0
\end{array}\right.
$$

Example 2. In the case when all three angles between the branches are equal, that is $\alpha=\beta=\gamma=\frac{2 \pi}{3}$ we have the following set of six matching conditions

$$
\left\{\begin{array}{l}
u_{1}(0)=u_{2}(0)=u_{3}(0), \\
u_{1}^{\prime}(0)+u_{2}^{\prime}(0)+u_{3}^{\prime}(0)=0, \\
u_{1}^{\prime \prime}(0)=u_{2}^{\prime \prime}(0)=u_{3}^{\prime \prime}(0), \\
\sum_{j=1}^{3} u_{j}^{\prime \prime \prime}(0)=0 .
\end{array}\right.
$$

Note that, the differential operator $L_{I V}$ with the matching conditions (4.3) is the square of the Laplace operator $L_{I I}$ subject to standard matching conditions (1.1). The only other cases for which $L_{I V}=L_{I I}^{2}$ are when $L_{I V}$ is accompanied with the matching conditions $u_{i}(0)=u_{i}^{\prime \prime}(0)=0$ or $u_{i}^{\prime}(0)=u_{i}^{\prime \prime \prime}(0)=0$ for $i=1,2,3$, or any combination of these conditions. The corresponding $L_{I I}$ operator satisfy the matching conditions $u_{i}(0)=0$ and $u_{i}^{\prime}(0)=0$ respectively.

The operator $L_{I V}$ with vertex conditions (4.3) is the only operator from the described family, which is a square of the Laplacian. It is easy to see that the vertex conditions corresponding to square of a Laplacian connect together separately the values of the functions and first derivatives on one hand and values of the second and third derivatives on the other hand. Moreover the conditions should be the same. Comparing the first and third lines in (2.9) we conclude that the $\sin \alpha=\sin \beta=\sin \gamma$. It follows that $\alpha=\beta=\gamma=2 \pi / 3$, since $\alpha+\beta+\gamma=2 \pi$ and $\alpha \neq \pi, \beta \neq \pi, \gamma \neq \pi$.

Example 3. In the degenerate case $\alpha=0$ and $\beta=\gamma=\pi$ we need to repeat the whole analysis. The tangential plane exists only if the function is continuous and the first derivatives are the same if taken in the same direction

$$
\left\{\begin{array}{l}
u_{1}(0)=u_{2}(0)=u_{3}(0), \\
-u_{1}^{\prime}(0)=u_{2}^{\prime}(0)=u_{3}^{\prime}(0) .
\end{array}\right.
$$

Writing the quadratic form and determining the corresponding self-adjoint operator leads to vertex conditions (2.8). 


\section{Conclusions and perspectives}

We obtained vertex conditions that appear as natural counterpart of free conditions in the case where the beams form nontrivial angles in the equilibrium position. One may prove that these conditions appear when approximating of beams by narrow channels is carried out similar to [EP, EP1, EP2, G, GJ, KZ, KZ1]. It is straightforward to generalize obtained conditions to the case where the number of beams is greater than 3. It is even more interesting to understand what is going on when the beams in the equilibrium do not lie in the same plane.

\section{Acknowledgements}

The authors would like to thank A. Watson for inspiring discussions as well as providing reference $[\mathrm{M}]$. The work of PK was partially supported by the Swedish Research Council (Grant D0497301) and ZiF-Zentrum für interdisziplinäre Forschung, Bielefeld (Cooperation Group Discrete and continuous models in the theory of networks).

\section{References}

[BK] G. Berkolaiko and P. Kuchment, Introduction to quantum graphs. Mathematical Surveys and Monographs, Amer. Math. Soc., 186 Providence, RI, 2013. xiv+270 pp.

[EP] P. Exner and O. Post, A general approximation of quantum graph vertex couplings by scaled Schrödinger operators on thin branched manifolds, Comm. Math. Phys. 322 (2013), no. 1, 207-227.

[EP1] P. Exner and O. Post, Approximation of quantum graph vertex couplings by scaled Schrödinger operators on thin branched manifolds, J. Phys. A 42 (2009), no. 41, 415305.

[EP2] P. Exner and O. Post, Convergence of spectra of graph-like thin manifolds, J. Geom. Phys. 54 (2005), no. 1, 77-115.

[DN2] B. Dekoninck, S. Nicaise, The eigenvalue problem for networks of beams, Linear Algebra Appl. 314 (2000), no. 1-3, 165189.

[DN1] B. Dekoninck, S. Nicaise, Control of networks of Euler-Bernoulli beams, ESAIM Control Optim. Calc. Var. 4 (1999), 5781 (electronic). 
[DM] V.A. Derkach, M.M. Malamud, Generalized resolvents and the boundary value problems for Hermitian operators with gaps, J. Funct. Anal. 95 (1991), 195.

[G] D. Grieser, Spectra of graph neighborhoods and scattering, Proc. Lond. Math. Soc., (3) 97 (2008), no. 3, 718-752.

[GJ] D. Grieser and D. Jerison, Asymptotics of eigenfunctions on plane domains, Pacific J. Math., 240 (2009), no. 1, 109-133.

[K] P. Kurasov, Quantum Graphs: Spectral Theory and Inverse Problems, to appear.

[KE] P. Kurasov and M. Enerbäck, Aharonov-Bohm ring touching a quantum wire: how to model it and to solve the inverse problem, (English summary), Rep. Math. Phys., 68 (2011), no. 3, 271-287.

[KN] P. Kurasov and M. Nowaczyk, Geometric properties of quantum graphs and vertex scattering matrices, Opuscula Math., 30 (2010), no. 3, 295309.

[KZ] P. Kuchment and H. Zeng, Asymptotics of spectra of Neumann Laplacians in thin domains. Advances in differential equations and mathematical physics (Birmingham, AL, 2002), 199-213, Contemp. Math., 327 Amer. Math. Soc., Providence, RI, 2003.

[KZ1] P. Kuchment and H. Zeng, Convergence of spectra of mesoscopic systems collapsing onto a graph, J. Math. Anal. Appl. 258 (2001), no. 2, 671-700.

[LL] L.D. Landau, E.M. Lifshitz, Course of theoretical physics, Vol. 7 Theory of elasticity (Translated from the Russian by J. B. Sykes and W. H. Reid) Third edition, Pergamon Press, Oxford, 1986.

[M] I. Mulins, Natural frequencies of tree shaped graphs, Master Thesis (11MAD300 - MMath), Loughborough Univ., 2012.

[P] O. Post, Spectral analysis on graph-like spaces, Lecture Notes in Mathematics 2039 (2012).

[RS] M. Reed, B. Simon, Methods of modern mathematical physics. II. Fourier analysis, self-adjointness, Academic Press, New York-London, 1975. 
[Y] D. R. Yafaev, Spectral and scattering theory of fourth order differential operators, Trans. AMS, Series 2, volume 225 (2008), viii+299. 